\title{
Stability estimate and regularization for a radially symmetric inverse heat conduction problem
}

\section{Wei Cheng*}

\section{"Correspondence:} chwei2007@163.com College of Science, Henan University of Technology, Zhengzhou, 450001, P.R. China

\begin{abstract}
This paper investigates a radially symmetric inverse heat conduction problem, which determines the internal surface temperature distribution of the hollow sphere from measured data at the fixed location inside it. This is an inverse and ill-posed problem. A conditional stability estimate is given on its solution by using Hölder's inequality. A wavelet regularization method is proposed to recover the stability of solution, and the technique is based on the dual least squares method and Shannon wavelet. A quite sharp error estimate between the approximate solution and the exact ones is obtained by choosing a suitable regularization parameter.
\end{abstract}

MSC: 65M30; 35R30; 35R25

Keywords: inverse heat conduction; ill-posed problems; stability estimate; regularization; error estimate

\section{Introduction}

A physical model considered here is a hollow sphere, and $R$ and $r_{0}$ denote its external and internal radius, respectively. Let the hollow sphere be adiabatic at its external surface, and a thermocouple is installed inside the hollow sphere at the radius $r=r_{1}, r_{0}<r_{1}<R$, as illustrated in Figure 1. Assuming a spherically symmetric temperature distribution of the model, the correspondingly mathematical model can be described as the following radially symmetric heat conduction problem:

$$
\begin{aligned}
& u_{t}=u_{r r}+\frac{2}{r} u_{r}, \quad r_{0}<r<R, t>0, \\
& u(r, 0)=0, \quad r_{0} \leq r \leq R, \\
& u\left(r_{1}, t\right)=g(t), \quad t \geq 0, \\
& u_{r}(R, t)=0, \quad t \geq 0,
\end{aligned}
$$

where $r$ denotes the radial coordinate, $g(t)$ is the temperature history at one fixed radius $r_{1}, r_{0}<r_{1}<R$. We want to recover the temperature distribution $u(r, \cdot)\left(r_{0} \leq r<r_{1}\right)$ based on the measured data of $g(\cdot)$. This is an inverse heat conduction problem.

The inverse heat conduction problem (IHCP) has numerous important applications in various sciences and engineering [1]. For example, determination of thermal fields at sur- 


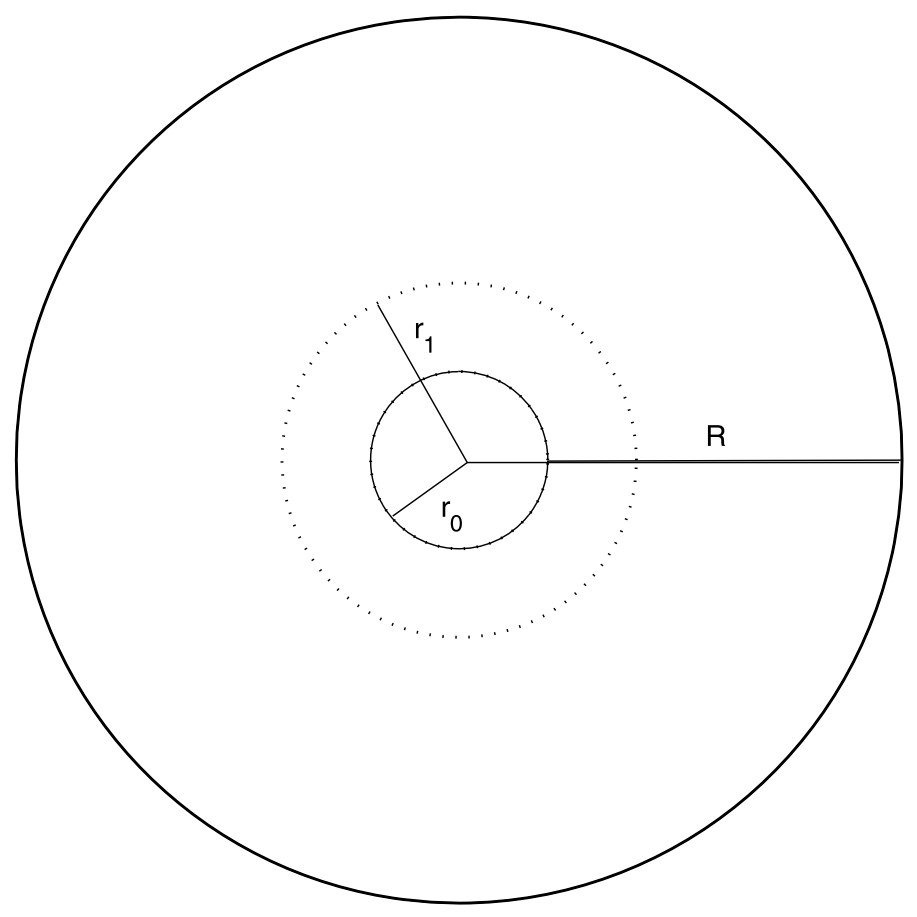

Figure 1 Physical model.

faces without access, obtaining the force applied to a complex structure from knowledge of the response and transfer function which describes the system, or the diagnosis of a disease by computerized tomography [2]. In all cases, the boundary conditions of these problems are inaccessible to measurements or not known. Usually sensors are installed beneath the surface and the unknown boundary conditions of these problems are estimated.

The solutions of inverse heat conduction problems (IHCPs) are very challenging, because IHCPs are severely ill-posed in the Hadamard sense that the solution (if it exists) does not depend continuously on the given data, i.e., a small measurement error in the given data can cause an enormous error in the solution [3, 4]. Therefore, an appropriate regularization method needs to be applied. These methods include the filtering method [5], the spectral method [6], the mollification method [7, 8], the boundary element method [9], the fundamental solution method [10], the wavelet and wavelet-Galerkin method [11-14], the Fourier method [15], the differential-difference method [16], the global time method [17], the modified Tikhonov method [18], the iterative method [19] etc. However, the results already published in the works on IHCP are mainly devoted to the heat equation with constant coefficient. The works presented for heat equation with variable coefficient are still limited. A few works have been developed for the inverse problems on heat equation with variable coefficient [20-23]. In [20], Fu used a simplified Tikhonov and a Fourier regularization methods to deal with an IHCP on heat equation with variable coefficient and provided two kinds of convergence rates. Grabski et al. [21] applied the method of fundamental solutions for identifying a time-dependent perfusion coefficient in the bioheat equation. A non-iterative inverse determination of temperature-dependent thermal conductivity was solved by Mierzwiczak and Kolodziej [22]. Chang and Chang [23] inves- 
tigated the determination of spatially- and temperature- dependent thermal conductivity by a semi-discretization method. In this work, we will use a wavelet method to deal with IHCP (1.1) $\left(r_{0} \leq r<r_{1}\right)$ with variable coefficient and to obtain a quite sharp error estimate between the approximate solution and the exact solution.

The wavelet method has become a powerful method for solving partial differential equations (PDEs). And the method has been applied to direct problems as well as to various types of inverse problems such as the IHCP [24], the Cauchy problem of Laplace equation [25, 26], the backward heat conduction problem [27], the inverse source identification problems [28, 29] and the Cauchy problem for the modified Helmholtz equation [30, 31]. It is worth mentioning that Feng and Ning [32] used a Meyer wavelet regularization method for solving numerical analytic continuation and presented the Hölder-type stability estimates. In this paper, we solve the radially symmetric inverse heat conduction problem (1.1) in the interval $\left[r_{0}, r_{1}\right)$ by determining the temperature distribution using a wavelet dual least squares method generated by the family of Shannon wavelets.

When we deal with problem $(1.1)$ in $L^{2}(\mathbb{R})$ with respect to variable $t$, we extend all functions of variable $t$ appearing in the paper to be zero for $t<0$. Since the measurement data of $g(t)$ contain noises, the solutions have to be sought from the data function $g^{\delta}(t) \in L^{2}(\mathbb{R})$, which satisfy

$$
\left\|g-g^{\delta}\right\| \leq \delta
$$

where the constant $\delta>0$ denotes a bound on the measurement error, and $\|\cdot\|$ represents the $L^{2}(\mathbb{R})$ norm. It is also assumed that there exists an a priori bound for function $u\left(r_{0}, t\right)$

$$
\left\|u\left(r_{0}, \cdot\right)\right\|_{H^{p}} \leq E, \quad p \geq 0
$$

where $\left\|u\left(r_{0}, \cdot\right)\right\|_{H^{p}}$ denotes the norm in the Sobolev space $H^{p}(\mathbb{R})$ defined by

$$
\left\|u\left(r_{0}, \cdot\right)\right\|_{H^{p}}:=\left(\int_{-\infty}^{\infty}\left(1+\xi^{2}\right)^{p}|\hat{f}(\xi)|^{2} d \xi\right)^{\frac{1}{2}}
$$

Using the Fourier transform with respect to the variable $t$, problem (1.1) can be formulated in a frequency space as follows:

$$
\left\{\begin{array}{l}
i \xi \hat{u}(r, \xi)=\frac{\partial^{2} \hat{u}(r, \xi)}{\partial r^{2}}+\frac{2}{r} \frac{\partial \hat{u}(r, \xi)}{\partial r}, \quad r \in\left(r_{0}, R\right], \xi \in \mathbb{R} \\
\hat{u}\left(r_{1}, \xi\right)=\hat{g}(\xi), \quad \xi \in \mathbb{R} \\
\hat{u}_{r}(R, \xi)=0, \quad \xi \in \mathbb{R} .
\end{array}\right.
$$

We can get a formal solution for problem (1.4), refer to [33],

$$
\hat{u}(r, \xi)=\left(r_{1} / r\right) \varphi(r, \xi) e^{\left(r_{1}-r\right) \sqrt{i \xi}} \hat{g}(\xi), \quad r \in\left[r_{0}, R\right), \xi \in \mathbb{R},
$$

where

$$
\varphi(r, \xi)=\frac{(\sqrt{i \xi} R+1) e^{2 r \sqrt{i \xi}}+(\sqrt{i \xi} R-1) e^{2 R \sqrt{i \xi}}}{(\sqrt{i \xi} R+1) e^{2 r 1 \sqrt{i \xi}}+(\sqrt{i \xi} R-1) e^{2 R \sqrt{i \xi}}} .
$$


According to Lemma 2.3 in [33], function $\varphi(r, \xi)$ satisfies

$$
c_{1} \leq|\varphi(r, \xi)| \leq c_{2}, \quad r \in\left[r_{0}, r_{1}\right), \xi \in \mathbb{R}
$$

where $c_{1}$ and $c_{2}$ are positive constants. Due to $\left|\left(r_{1} / r\right) \varphi(r, \xi) e^{\left(r_{1}-r\right) \sqrt{i \xi}}\right|$ increases rapidly with exponential order as $|\xi| \rightarrow \infty$, the Fourier transform of the exact data $g(t)$ must decay rapidly at high frequencies for $r_{1}>r$. But such a decay is not likely to occur in $g^{\delta}(t)$. So, a small measurement error in the given data $g^{\delta}(t)$ in high frequency components can completely destroy the solution of problem (1.1) for $r \in\left[r_{0}, r_{1}\right)$.

For problem (1.1), we define an operator $A_{r}: u(r, \cdot) \longmapsto g(\cdot)$ in the space $X=L^{2}(\mathbb{R})$. Then problem (1.1) can be rewritten as

$$
A_{r} u(r, t)=g(t), \quad \forall u(r, \cdot) \in X, r_{0} \leq r<r_{1} .
$$

According to expression (1.5), there holds

$$
\widehat{A_{r} u}(r, \xi)=\hat{g}(\xi)=\left(r / r_{1}\right) e^{\left(r-r_{1}\right) \sqrt{i \xi}} \varphi^{-1}(r, \xi) \hat{u}(r, \xi), \quad r \in\left[r_{0}, r_{1}\right) .
$$

Then we have $\widehat{A_{r} u(r, \xi)}:=\widehat{A_{r}} \hat{u}(r, \xi)$ and a multiplication operator $\widehat{A_{r}}: L^{2}(\mathbb{R}) \longmapsto L^{2}(\mathbb{R})$ given by

$$
\widehat{A_{r}} \hat{u}(r, \xi)=\left(r / r_{1}\right) e^{\left(r-r_{1}\right) \sqrt{i \xi}} \varphi^{-1}(r, \xi) \hat{u}(r, \xi)
$$

Therefore, we have the following lemma.

Lemma 1.1 If $A_{r}^{*}$ is the adjoint of $A_{r}$, then $A_{r}^{*}$ corresponds to the following problem:

$$
\left\{\begin{array}{l}
-\frac{\partial U}{\partial t}=\frac{\partial^{2} U}{\partial r^{2}}+\frac{2}{r} \frac{\partial U}{\partial r}, \quad r_{0}<r \leq R, t \geq 0 \\
U(r, 0)=0, \quad r_{0} \leq r \leq R \\
U\left(r_{1}, t\right)=g(t), \quad t \geq 0 \\
U_{r}(R, t)=0, \quad t \geq 0
\end{array}\right.
$$

and

$$
\widehat{A_{r}^{*}}=\left(r / r_{1}\right) e^{\left(r-r_{1}\right) \sqrt{i \xi}} \overline{\varphi^{-1}(r, \xi)}
$$

Proof Using the following relations and expression (1.9)

$$
\left\langle A_{r} u, v\right\rangle=\left\langle\widehat{A_{r}} \hat{u}, \hat{v}\right\rangle=\left\langle\hat{u}, \widehat{A}_{r}^{*} \hat{v}\right\rangle=\left\langle u, A_{r}^{*} v\right\rangle=\left\langle\hat{u}, \widehat{A_{r}^{*}} \hat{v}\right\rangle
$$

where $\langle\cdot, \cdot\rangle$ denotes the inner product, we can obtain the adjoint operator $A_{r}^{*}$ of $A_{r}$ in the frequency domain

$$
\widehat{A_{r}^{*}}=\widehat{A}_{r}^{*}=\left(r / r_{1}\right) e^{\left(r-r_{1}\right) \overline{\sqrt{i \xi}}} \overline{\varphi^{-1}(r, \xi)} .
$$


Applying the Fourier transform with respect to the variable $t$, we can rewrite problem (1.10) in the following form (in the frequency space):

$$
\left\{\begin{array}{l}
-i \xi \hat{U}(r, \xi)=\frac{\partial^{2} \hat{U}(r, \xi)}{\partial r^{2}}+\frac{2}{r} \frac{\partial \hat{U}(r, \xi)}{\partial r}, \quad r \in\left(r_{0}, R\right], \xi \in \mathbb{R} \\
\hat{U}\left(r_{1}, \xi\right)=\hat{g}(\xi), \quad \xi \in \mathbb{R} \\
\left|\hat{U}_{r}(R, \xi)\right|=0, \quad \xi \in \mathbb{R} .
\end{array}\right.
$$

Taking the conjugate operator for problem (1.4), we know that $\hat{U}(r, \xi)=\overline{\hat{u}(r, \xi)}$. So, combining with (1.5), we get

$$
\hat{U}(r, \xi)=\overline{\hat{u}(r, \xi)}=\left(r_{1} / r\right) e^{\left(r_{1}-r\right) \sqrt{i \xi}} \overline{\varphi(r, \xi)} \hat{g}(\xi)
$$

and

$$
\hat{g}(\xi)=\left(r / r_{1}\right) e^{\left(r-r_{1}\right) \sqrt{i \xi}} \overline{\varphi^{-1}(r, \xi)} \hat{U}(r, \xi)=\widehat{A_{r}^{*}} \hat{U}(r, \xi)=\widehat{A_{r}^{*} U} .
$$

The outline of the paper is as follows. In Section 2, using Hölder's inequality, we prove the conditional stability for IHCP (1.1) in the interval $\left[r_{0}, r_{1}\right)$. The relevant properties of Shannon wavelets are summarized in Section 3. The last section presents error estimates via wavelet dual least squares method approximation.

\section{A conditional stability estimate}

In this section, we give a conditional stability in the following theorem.

Theorem 2.1 Let $u(r, t)$ be the exact solution of problem (1.1) given by (1.5) and the a priori bound (1.3) hold. Then, for a fixed $r \in\left(r_{0}, r_{1}\right)$, we have the following estimate:

$$
\|u(r, \cdot)\| \leq c_{2}\left(c_{1}\right)^{\frac{r-r_{1}}{r_{1}-r_{0}}}\left(r_{1} / r_{0}\right)^{\frac{r-r_{0}}{r_{1}-r_{0}}}\left\|u\left(r_{0}, \cdot\right)\right\|^{\frac{r_{1}-r}{r_{1}-r_{0}}}\|g\|^{\frac{r-r_{0}}{r_{1}-r_{0}}}
$$

where $c_{1}$ and $c_{2}$ are constants given by (1.6).

Proof Using Parseval's formula, expression (1.5) and Hölder's inequality, we have

$$
\begin{aligned}
\|u(r, \cdot)\|^{2} & =\|\hat{u}(r, \cdot)\|^{2}=\int_{-\infty}^{\infty}\left|\left(r_{1} / r\right) \varphi(r, \xi) e^{\left(r_{1}-r\right) \sqrt{i \xi}} \hat{g}(\xi)\right|^{2} d \xi \\
& =\int_{-\infty}^{\infty}\left[\left|\left(r_{1} / r\right) \varphi(r, \xi)\right|^{\frac{2\left(r_{1}-r_{0}\right)}{r_{1}-r}}\left|e^{\left(r_{1}-r_{0}\right) \sqrt{i \xi}} \hat{g}(\xi)\right|^{2}\right]^{\frac{r_{1}-r}{r_{1}-r_{0}}}\left[|\hat{g}(\xi)|^{2}\right]^{\frac{r-r_{0}}{r_{1}-r_{0}}} d \xi \\
& \leq\left(\int_{-\infty}^{\infty}\left|\left(r_{1} / r\right) \varphi(r, \xi)\right|^{\frac{2\left(r_{1}-r_{0}\right)}{r_{1}-r}}\left|e^{\left(r_{1}-r_{0}\right) \sqrt{i \xi}} \hat{g}(\xi)\right|^{2} d \xi\right)^{\frac{r_{1}-r}{r_{1}-r_{0}}}\left(\int_{-\infty}^{\infty}|\hat{g}(\xi)|^{2} d \xi\right)^{\frac{r-r_{0}}{r_{1}-r_{0}}} \\
& =\left(\int_{-\infty}^{\infty}\left|\left(r_{1} / r\right) \varphi(r, \xi)\right|^{\frac{2\left(r_{1}-r_{0}\right)}{r_{1}-r}}\left|\left(r_{1} / r_{0}\right) \varphi\left(r_{0}, \xi\right)\right|^{-2}\left|\hat{u}\left(r_{0}, \xi\right)\right|^{2} d \xi\right)^{\frac{r_{1}-r}{r_{1}-r_{0}}}\|g\|^{\frac{2\left(r-r_{0}\right)}{r_{1}-r_{0}}} \\
& \leq \sup _{\xi \in \mathbb{R}}\left[\left|\left(r_{1} / r\right) \varphi(r, \xi)\right|^{2}\left|\left(r_{1} / r_{0}\right) \varphi\left(r_{0}, \xi\right)\right|^{\frac{2\left(r-r_{1}\right)}{r_{1}-r_{0}}}\right]\left\|\hat{u}\left(r_{0}, \cdot\right)\right\|^{\frac{2\left(r_{1}-r\right)}{r_{1}-r_{0}}}\|g\|^{\frac{2\left(r-r_{0}\right)}{r_{1}-r_{0}}} .
\end{aligned}
$$


From inequalities (1.6), we get

$$
\left|\left(r_{1} / r\right) \varphi(r, \xi)\right|^{2}\left|\left(r_{1} / r_{0}\right) \varphi\left(r_{0}, \xi\right)\right|^{\frac{2\left(r-r_{1}\right)}{r_{1}-r_{0}}} \leq\left|\left(r_{1} / r\right) c_{2}\right|^{2}\left|\left(r_{1} / r_{0}\right) c_{1}\right|^{\frac{2\left(r-r_{1}\right)}{r_{1}-r_{0}}}
$$

Then there holds

$$
\|u(r, \cdot)\|^{2} \leq c_{2}^{2}\left(c_{1}\right)^{\frac{2\left(r-r_{1}\right)}{r_{1}-r_{0}}}\left(r_{1} / r_{0}\right)^{\frac{2\left(r-r_{0}\right)}{r_{1}-r_{0}}}\left\|u\left(r_{0}, \cdot\right)\right\|^{\frac{2\left(r_{1}-r\right)}{r_{1}-r_{0}}}\|g\|^{\frac{2\left(r-r_{0}\right)}{r_{1}-r_{0}}} .
$$

The conclusion of Theorem 2.1 is proved.

Remark 2.2 If $u_{1}(r, t)$ and $u_{2}(r, t)$ are the solutions of problem (1.1) with the exact data $g_{1}(t)$ and $g_{2}(t)$, respectively, then for a fixed $r \in\left(r_{0}, r_{1}\right)$, there holds

$$
\left\|u_{1}(r, \cdot)-u_{2}(r, \cdot)\right\| \leq C\left\|u_{1}\left(r_{0}, \cdot\right)-u_{2}\left(r_{0}, \cdot\right)\right\|^{\frac{r_{1}-r}{r_{1}-r_{0}}}\left\|g_{1}(\cdot)-g_{1}(\cdot)\right\|^{\frac{r-r_{0}}{r_{1}-r_{0}}},
$$

where $C=c_{2}\left(c_{1}\right)^{\frac{r-r_{1}}{r_{1}-r_{0}}}\left(r_{1} / r_{0}\right)^{\frac{r-r_{0}}{r_{1}-r_{0}}}$. It is obvious that if $\left\|g_{1}(\cdot)-g_{1}(\cdot)\right\| \rightarrow 0$, then $\| u_{1}(r, \cdot)-$ $u_{2}(r, \cdot) \|$ for $r_{0}<r \leq r_{1}$.

In the next section, the relevant properties of Shannon wavelets are summarized.

\section{The Shannon wavelets}

Suppose that $\phi$ and $\psi$ are the Shannon scaling and wavelet functions whose Fourier transforms are given by

$$
\hat{\phi}(\xi)= \begin{cases}1, & |\xi| \leq \pi \\ 0, & \text { otherwise }\end{cases}
$$

and

$$
\hat{\psi}(\xi)= \begin{cases}e^{-i \frac{\xi}{2}}, & \pi \leq|\xi| \leq 2 \pi \\ 0, & \text { otherwise }\end{cases}
$$

Let $\phi_{j, k}(t):=2^{\frac{j}{2}} \phi\left(2^{j} t-k\right), \psi_{j, k}(t):=2^{\frac{j}{2}} \psi\left(2^{j} t-k\right), j, k \in \mathbb{Z}, \Psi_{-1, k}:=\phi_{0, k}$ and $\Psi_{l, k}:=\psi_{l, k}$ for $l \geq 0$, the index set

$$
\begin{aligned}
& \tilde{I}=\{\{j, k\}: j, k \in \mathbb{Z}\} \subset \mathbb{Z}^{2}, \\
& \tilde{I}_{J}=\{\{j, k\}: j=-1,0, \ldots, J-1 ; k \in \mathbb{Z}\} \subset \mathbb{Z}^{2} .
\end{aligned}
$$

Then the subspaces $V_{J}$ can be defined

$$
V_{J}=\overline{\operatorname{span}\left\{\Psi_{\lambda}\right\}_{\lambda \in \tilde{I}_{J}}}
$$

and an orthogonal projection $P_{J}: L^{2}(\mathbb{R}) \longmapsto V_{J}$ :

$$
P_{J} \varphi=\sum_{\lambda \in \tilde{I}_{J}}\left\langle\varphi, \Psi_{\lambda}\right\rangle \Psi_{\lambda}, \quad \forall \varphi \in L^{2}(\mathbb{R})
$$


We have, for any $k \in \mathbb{Z}$,

$$
\begin{aligned}
& \operatorname{supp}\left(\hat{\psi}_{j, k}\right)=\left\{\xi: \pi 2^{j} \leq|\xi| \leq \pi 2^{j+1}\right\} \\
& \operatorname{supp}\left(\hat{\phi}_{j, k}\right)=\left\{\xi:|\xi| \leq \pi 2^{j}\right\}
\end{aligned}
$$

Expression (3.4) shows that $P_{J}$ can be considered as a low pass filter.

\section{Regularization and error estimates}

In this section, the wavelet dual least squares method will be described and error estimates be given by Theorems 4.1-4.3.

\subsection{Dual least squares method}

We now introduce the dual least squares method for approximation of the solutions of problem (1.1). For the operator equation $A u=g$, a general projection method is generated by two subspace families $\left\{V_{j}\right\}$ and $\left\{Y_{j}\right\}$ of $X$. Then the approximate solution $u_{j} \in V_{j}$ is defined to be the solution of the following problem:

$$
\left\langle A u_{j}, y\right\rangle=\langle g, y\rangle, \quad \forall y \in Y_{j} .
$$

If $V_{j} \subset R\left(A^{*}\right)$ and subspaces $Y_{j}$ are chosen in such a way that

$$
A^{*} Y_{j}=V_{j}
$$

then there is a special case of projection method known as the dual least squares method. Suppose that $\left\{\psi_{\lambda}\right\}_{\lambda \in \tilde{I}_{j}}$ is an orthogonal basis of $V_{j}$ and $y_{\lambda}$ is the solution of the equation

$$
A^{*} y_{\lambda}=k_{\lambda} \psi_{\lambda}, \quad\left\|y_{\lambda}\right\|=1
$$

Then we can obtain the approximate solution

$$
u_{j}=\sum_{\lambda \in \tilde{I}_{j}}\left\langle g, y_{\lambda}\right\rangle \frac{1}{k_{\lambda}} \psi_{\lambda} .
$$

According to (4.3), we easily conclude $u_{J}=P_{J} u$. In order to give an error estimate for the regularized solution, we need a sequence of subspaces $Y_{j}$ approximating the space $X$ and contained in the range of $A^{*}$. From $A^{*} Y_{j}=V_{j}$, the subspaces $Y_{j}$ are spanned by $w_{\lambda}, \lambda \in \tilde{I}_{J}$, where

$$
A^{*} w_{\lambda}=\Psi_{\lambda} \quad \text { and } \quad k_{\lambda}=\left\|w_{\lambda}\right\|^{-1}, \quad y_{\lambda}=\frac{w_{\lambda}}{\left\|w_{\lambda}\right\|}=k_{\lambda} w_{\lambda} .
$$

We know that $w_{\lambda}$ is a solution of the following problem (see Lemma 1.1):

$$
\left\{\begin{array}{l}
-\frac{\partial U}{\partial t}=\frac{\partial^{2} U}{\partial r^{2}}+\frac{2}{r} \frac{\partial U}{\partial r}, \quad r_{0}<r \leq R, t \geq 0 \\
U(r, 0)=0, \quad r_{0} \leq r \leq R \\
U\left(r_{1}, t\right)=\Psi_{j, k}(t), \quad t \geq 0 \\
U_{r}(R, t)=0, \quad t \geq 0
\end{array}\right.
$$


Because supp $\hat{\psi}_{j, k}$ is compact, the solution exists for any $t \in(0, \infty)$. Analogously, the solution of the adjoint equation is unique. So, for given $\Psi_{\lambda}, w_{\lambda}$ can be uniquely determined according to (4.5). From (1.13), there holds

$$
\hat{w}_{\lambda}=\frac{r_{1}}{r} e^{\left(r_{1}-r\right) \sqrt{\sqrt{i \xi}}} \overline{\varphi(r, \xi)} \hat{\Psi}_{\lambda}(\xi), \quad \lambda=\{j, k\}
$$

combining with (4.4), we have

$$
\hat{y}_{\lambda}=\frac{r_{1}}{r} e^{\left(r_{1}-r\right) \sqrt{\sqrt{i \xi}}} \overline{\varphi(r, \xi)} k_{\lambda} \hat{\Psi}_{\lambda}(\xi), \quad \lambda=\{j, k\}
$$

Thus we have the approximate solution for noisy data $g^{\delta}$ given by

$$
P_{J} u^{\delta}(r, t)=u_{J}^{\delta}=\sum_{\lambda \in \tilde{I}_{J}}\left|u^{\delta}, \Psi_{\lambda}\right| \Psi_{\lambda}=\sum_{\lambda \in \tilde{I}_{J}}\left|g^{\delta}, y_{\lambda}\right\rangle \frac{1}{k_{\lambda}} \Psi_{\lambda} .
$$

\subsection{Error estimates}

We estimate firstly the errors $\left\|P_{J} u-P_{J} u^{\delta}\right\|$ and $\left\|u-P_{J} u\right\|$ by Theorems 4.1 and 4.2 , respectively.

Theorem 4.1 (Stability) Let $P_{J} u(r, t)$ given by (4.3) and $P_{J} u^{\delta}(r, t)$ given by $(4.8)$ be the regularized approximate solutions to $u(r, t)$ for the data $g$ and $g^{\delta}$, respectively. If the measured data $g^{\delta}(t)$ satisfy condition (1.2), then for any fixed $r \in\left[r_{0}, r_{1}\right)$, we have

$$
\left\|P_{J} u-P_{J} u^{\delta}\right\| \leq\left(c_{2} r_{1} / r_{0}\right) e^{\left(r_{1}-r\right) \sqrt{\frac{1}{2} \pi 2^{J}}} \delta .
$$

Proof Due to (4.3), (4.8) and (4.7), for any fixed $r \in\left[r_{0}, r_{1}\right)$, there holds

$$
\begin{aligned}
& \left\|P_{J} u(r, \cdot)-P_{J} u^{\delta}(r, \cdot)\right\|=\left\|\sum_{\lambda \in \tilde{I}_{J}}\left\langle g-g^{\delta}, y_{\lambda}\right\rangle \frac{1}{k_{\lambda}} \Psi_{\lambda}\right\| \\
& =\left\|\sum_{\lambda \in \tilde{I}_{I}}\left\langle\hat{g}-\widehat{g^{\delta}}, \hat{y}_{\lambda}\right\rangle \frac{1}{k_{\lambda}} \hat{\Psi}_{\lambda}\right\| \\
& =\| \sum_{\lambda \in \tilde{I}_{J}}\left\langle\hat{g}-\widehat{g^{\delta}}, \frac{r_{1}}{r} e^{\left(r_{1}-r\right) \overline{\sqrt{i \xi}}} \overline{\varphi(r, \xi)} k_{\lambda} \hat{\Psi}_{\lambda}\right| \frac{1}{k_{\lambda}} \hat{\Psi}_{\lambda} \| \\
& \leq \sup _{\pi 2^{J-1} \leq|\xi| \leq \pi 2^{J}}\left|\left(r_{1} / r\right) e^{\left(r_{1}-r\right) \sqrt{\sqrt{\xi}}} \overline{\varphi(r, \xi)}\right| \cdot \| \sum_{\lambda \in \tilde{I}_{J}}\left|\hat{g}-\widehat{g^{\delta}}, \hat{\Psi}_{\lambda}\right\rangle \hat{\Psi}_{\lambda} \| \\
& \leq \sup _{\pi 2^{J-1} \leq|\xi| \leq \pi 2^{J}}\left|\left(r_{1} / r\right) e^{\left(r_{1}-r\right) \sqrt{i \xi}} \varphi(r, \xi)\right| \cdot\left\|\hat{P}_{J}\left(\hat{g}-\widehat{g^{\delta}}\right)\right\|,
\end{aligned}
$$

combining with inequality (1.6) and condition (1.2),

$$
\begin{aligned}
\left\|P_{J} u(r, \cdot)-P_{J} u^{\delta}(r, \cdot)\right\| & \leq\left(c_{2} r_{1} / r_{0}\right) \sup _{\pi 2^{J-1} \leq|\xi| \leq \pi 2^{J}} e^{\left(r_{1}-r\right) \sqrt{|\xi| / 2}} \delta \\
& \leq\left(c_{2} r_{1} / r_{0}\right) e^{\left(r_{1}-r\right) \sqrt{\frac{1}{2} \pi 2^{J}}} \delta .
\end{aligned}
$$


Theorem 4.2 (Convergence) If $u(r, t)$ is the solution of problem (1.1) satisfying the a priori condition (1.3), then for any fixed $r \in\left[r_{0}, r_{1}\right)$, we have

$$
\left\|u(r, \cdot)-P_{J} u(r, \cdot)\right\| \leq\left(c_{2} / c_{1}\right)\left(2^{J+1}\right)^{-p} e^{\left(r_{0}-r\right) \sqrt{\frac{1}{2} \pi 2^{2}}} E .
$$

Proof According to (3.4), we get

$$
\begin{aligned}
& u(r, \cdot)=\sum_{\lambda}\left\langle u(r, \cdot), \Psi_{\lambda}\right| \Psi_{\lambda}, \\
& P_{J} u(r, \cdot)=\sum_{\lambda \in \tilde{I}_{J}}\left\langle u(r, \cdot), \Psi_{\lambda}\right| \Psi_{\lambda} .
\end{aligned}
$$

By using Parseval's relation and (1.5), (1.6), (1.3), there holds

$$
\begin{aligned}
& \left\|u(r, \cdot)-P_{J} u(r, \cdot)\right\| \\
& =\left\|\hat{u}(r, \cdot)-\widehat{P_{J} u}(r, \cdot)\right\|=\left\|\sum_{\lambda \in \tilde{I}}\left\langle\hat{u}, \hat{\Psi}_{\lambda}\right\rangle \hat{\Psi}_{\lambda}-\sum_{\lambda \in \tilde{I}_{J}}\left\langle\hat{u}, \hat{\Psi}_{\lambda}\right\rangle \hat{\Psi}_{\lambda}\right\| \\
& =\left\|\sum_{\lambda \in \tilde{I}_{j \geq J+1}}\left\langle\hat{u}, \hat{\Psi}_{\lambda}\right\rangle \hat{\Psi}_{\lambda}\right\|=\left\|\sum_{\lambda \in \tilde{I}_{j \supset J+1}}\left\langle\left(r_{1} / r\right) \varphi(r, \cdot) e^{\left(r_{1}-r\right) \sqrt{i(\cdot)}} \hat{g}(\cdot), \hat{\Psi}_{\lambda}\right\rangle \hat{\Psi}_{\lambda}\right\| \\
& =\left\|\sum_{\lambda \in \tilde{I}_{j \supset J+1}}\left\langle\left(r_{0} / r\right) \varphi(r, \cdot) \varphi^{-1}\left(r_{0}, \cdot\right) e^{\left(r_{0}-r\right) \sqrt{i(\cdot)}} \hat{u}\left(r_{0}, \cdot\right), \hat{\Psi}_{\lambda}\right) \hat{\Psi}_{\lambda}\right\| \\
& \leq \sup _{\pi 2^{J} \leq|\xi| \leq \pi 2^{J+1}}\left|\frac{r_{0} \varphi(r, \xi)}{r \varphi\left(r_{0}, \xi\right)} e^{\left(r_{0}-r\right) \sqrt{i \xi}|\xi|^{-p}}\right| \mid \sum_{\lambda \in \tilde{I}_{j \geq J+1}}\left\langle\left(1+(\cdot)^{2}\right)^{p / 2} \hat{u}\left(r_{0}, \cdot\right), \hat{\Psi}_{\lambda}\right| \hat{\Psi}_{\lambda} \| \\
& \leq \sup _{\pi 2^{J} \leq|\xi| \leq \pi 2^{J+1}}\left(c_{2} / c_{1}\right)|\xi|^{-p} e^{\left(r_{0}-r\right) \sqrt{|\xi| / 2}} E \leq\left(c_{2} / c_{1}\right)\left(2^{J+1}\right)^{-p} e^{\left(r_{0}-r\right) \sqrt{\frac{1}{2} \pi 2^{J}}} E .
\end{aligned}
$$

We have proved estimate (4.10).

Theorem 4.3 Let $u(r, t)$ be the exact solution of $(1.1)$ and $P_{J} u^{\delta}$ given by $(4.8)$ be the regularized approximate solution to $u(r, t)$. If the measured data $g^{\delta}(t)$ satisfies condition (1.2) and the a priori condition (1.3) is valid when we select

$$
J=\log _{2}\left[\frac{2}{\pi}\left(\frac{1}{r_{1}-r_{0}} \ln \left(\frac{E}{\delta}\left(\ln \frac{E}{\delta}\right)^{-2 p}\right)\right)^{2}\right]
$$

then for any fixed $r \in\left[r_{0}, r_{1}\right)$, we have

$$
\begin{aligned}
& \left\|u(r, \cdot)-P_{J} u^{\delta}(r, \cdot)\right\| \\
& \quad \leq E^{1-\frac{r-r_{0}}{r_{1}-r_{0}}} \delta^{\frac{r-r_{0}}{r_{1}-r_{0}}}\left(\ln \frac{E}{\delta}\right)^{-2 p\left(1-\frac{r-r_{0}}{r_{1}-r_{0}}\right)}(C+o(1)) \quad \text { for } \delta \rightarrow 0,
\end{aligned}
$$

where $C=\left(c_{2} / c_{1}\right)\left(r_{1}-r_{0}\right)^{2 p}+\left(c_{2} r_{1} / r_{0}\right)$. 
Proof From Theorem 4.1, Theorem 4.2 and the choice rule (4.11) of $J$, we can get

$$
\begin{aligned}
&\left\|u(r, \cdot)-P_{J} u^{\delta}(r, \cdot)\right\| \\
& \leq\left(c_{2} / c_{1}\right)\left(2^{J+1}\right)^{-p} e^{\left(r_{0}-r\right) \sqrt{\frac{1}{2} \pi 2^{l}}} E+\left(c_{2} r_{1} / r_{0}\right) e^{\left(r_{1}-r\right) \sqrt{\frac{1}{2} \pi 2^{2}}} \delta \\
& \leq\left(c_{2} / c_{1}\right) E\left(r_{1}-r_{0}\right)^{2 p}\left(\ln \left(\frac{E}{\delta}\left(\ln \frac{E}{\delta}\right)^{-2 p}\right)\right)^{-2 p}\left(\frac{E}{\delta}\left(\ln \frac{E}{\delta}\right)^{-2 p}\right)^{\frac{r_{0}-r}{r_{1}-r_{0}}} \\
&+\left(c_{2} r_{1} / r_{0}\right) \delta\left(\frac{E}{\delta}\left(\ln \frac{E}{\delta}\right)^{-2 p}\right)^{\frac{r_{1}-r}{r_{1}-r_{0}}} \\
& \leq E^{1-\frac{r-r_{0}}{r_{1}-r_{0}}} \delta^{\frac{r-r_{0}}{r_{1}-r_{0}}}\left(\ln \frac{E}{\delta}\right)^{-2 p\left(1-\frac{r-r_{0}}{r_{1}-r_{0}}\right)}\left\{\frac{c_{2}\left(\left(r_{1}-r_{0}\right) \ln \frac{E}{\delta}\right)^{2 p}}{c_{1}\left(\ln \left(\frac{E}{\delta}\left(\ln \frac{E}{\delta}\right)^{-2 p}\right)\right)^{2 p}}+\frac{c_{2} r_{1}}{r_{0}}\right\} .
\end{aligned}
$$

Note that

$$
\frac{\ln \frac{E}{\delta}}{\ln \left(\frac{E}{\delta}\left(\ln \frac{E}{\delta}\right)^{-2 p}\right)}=\frac{\ln \frac{E}{\delta}}{\ln \frac{E}{\delta}-2 p \ln \left(\ln \frac{E}{\delta}\right)} \rightarrow 1 \quad \text { for } \delta \rightarrow 0 .
$$

We have obtained estimate (4.12).

\section{Remark 4.4}

(i) If $p=0$ and $r_{0}<r<r_{1}$, estimate (4.12) becomes

$$
\left\|u(r, \cdot)-P_{J} u^{\delta}(r, \cdot)\right\| \leq\left(\left(c_{2} / c_{1}\right)+\left(c_{2} r_{1} / r_{0}\right)\right) E^{1-\frac{r-r_{0}}{r_{1}-r_{0}}} \delta^{\frac{r-r_{0}}{r_{1}-r_{0}}}
$$

which is a Hölder stability estimate.

(ii) If $p>0$, estimate (4.12) is a logarithmical-Hölder stability estimate, especially at $r=r_{0}$, it becomes

$$
\left\|u\left(r_{0}, \cdot\right)-u^{\delta}\left(r_{0}, \cdot\right)\right\| \leq E\left(\ln \frac{E}{\delta}\right)^{-2 p}(C+o(1)) \rightarrow 0 \quad \text { for } \delta \rightarrow 0,
$$

which is a logarithmical stability estimate.

\section{Conclusion}

In this paper the radially symmetric inverse heat conduction problem is considered. A conditional stability result is established by utilizing the a priori bound. We obtain a regularized solution by a wavelet dual least squares method and the error estimate of logarithmic Hölder type between the approximate solution and the exact ones by choosing a suitable regularization parameter.

\section{Abbreviation \\ IHCP, inverse heat conduction problem.}

Competing interests

The author declares that she has no competing interests. 


\section{Acknowledgements}

The author would like to thank the anonymous referees for their valuable comments and helpful suggestions on this work. The project is supported by the National Natural Science Foundation (NNSF) of China (11561045), the Natural Science Foundation of Henan Province of China (132300410231).

\section{Publisher's Note}

Springer Nature remains neutral with regard to jurisdictional claims in published maps and institutional affiliations.

\section{Received: 30 November 2016 Accepted: 4 April 2017 Published online: 18 April 2017}

\section{References}

1. Alifanov, OM: Inverse Heat Transfer Problems. Springer, Berlin (1994)

2. Fernandes, AP, Santos, MB, Guimarães, G: An analytical transfer function method to solve inverse heat conduction problems. Appl. Math. Model. 39,6897-6914 (2015)

3. Beck, JV, Blackwell, B, Clair, SR: Inverse Heat Conduction: IIl-Posed Problems. Wiley, New York (1985)

4. Carasso, A: Determining surface temperatures from interior observations. SIAM J. Appl. Math. 42, 558-574 (1982)

5. Seidman, T, Eldén, L: An optimal filtering method for the sideways heat equation. Inverse Probl. 6, 681-696 (1990)

6. Tautenhahn, U: Optimality for ill-posed problems under general source conductions. Numer. Funct. Anal. Optim. 19, 377-398 (1998)

7. Murio, DA: Stable numerical evaluation of Grünwald-Letnikov fractional derivatives applied to a fractional IHCP. Inverse Probl. Sci. Eng. 17(2), 229-243 (2009)

8. Garshasbi, M, Dastour, H: Estimation of unknown boundary functions in an inverse heat conduction problem using a mollified marching scheme. Numer. Algorithms 68(4), 769-790 (2015)

9. Hào, DN, Thanh, PX, Lesnic, D, Johansson, BT: A boundary element method for a multi-dimensional inverse heat conduction problem. Int. J. Comput. Math. 13, 1540-1554 (2012)

10. Hon, YC, Wei, T: A fundamental solution method for inverse heat conduction problem. Eng. Anal. Bound. Elem. 28, 489-495 (2004)

11. Eldén, L, Berntsson, F, Regińska, T: Wavelet and Fourier methods for solving the sideways heat equation. SIAM J. Sci. Comput. 21(6), 2187-2205 (2000)

12. Regińska, T, Eldén, L: Solving the sideways heat equation by a wavelet-Galerkin method. Inverse Probl. 13, 1093-1106 (1997)

13. Regińska, T, Eldén, L: Stability and convergence of wavelet-Galerkin method for the sideways heat equation. J. Inverse III-Posed Probl. 8, 31-49 (2000)

14. Fu, CL, Qiu, CY: Wavelet and error estimation of surface heat flux. J. Comput. Appl. Math. 150, 143-155 (2003)

15. Wróblewska, A, Frackowiak, A, Cialkowski, M: Regularization of the inverse heat conduction problem by the discrete Fourier transform. Inverse Probl. Sci. Eng. 24(2), 195-212 (2016)

16. Qian, Z, Zhang, Q: Differential-difference regularization for a 2D inverse heat conduction problem. Inverse Probl. 26(9), $095015(2010)$

17. Elkinsa, BS, Keyhania, M, Franke, Jl: Global time method for inverse heat conduction problem. Inverse Probl. Sci. Eng. 20(5), 651-664 (2012)

18. Xiong, XT, Hon, YC: Regularization error analysis on a one-dimensional inverse heat conduction problem in multilayer domain. Inverse Probl. Sci. Eng. 21(5), 865-887 (2013)

19. Chapko, R, Johansson, BT, Vavrychuk, V: A projected iterative method based on integral equations for inverse heat conduction in domains with a cut. Inverse Probl. 29(6), 065003 (2013)

20. Fu, CL: Simplified Tikhonov and Fourier regularization methods on a general sideways parabolic equation. J. Comput. Appl. Math. 167, 449-463 (2004)

21. Grabski, JK, Lesnic, D, Johansson, BT: Identification of a time-dependent perfusion coefficient in the bioheat equation. Int. Commun. Heat Mass Transf. 75, 218-222 (2016)

22. Mierzwiczak, M, Kolodziej, JA: The determination temperature-dependent thermal conductivity as inverse steady heat conduction problem. Int. J. Heat Mass Transf. 54, 790-796 (2011)

23. Chang, CL, Chang, M: Inverse determination of thermal conductivity using semi-discretization method. Appl. Math. Model. 33, 1644-1655 (2009)

24. Regińska, T: Application of wavelet shrinkage to solving the sideways heat equation. BIT Numer. Math. 41(5), 1101-1110 (2000)

25. Vani, C, Avudainayagam, A: Regularized solution of the Cauchy problem for the Laplace equation using Meyer Wavelets. Math. Comput. Model. 36, 1151-1159 (2002)

26. Qiu, CY, Fu, CL: Wavelets and regularization of the Cauchy problem for the Laplace equation. J. Math. Anal. Appl. 33(2), 1440-1447 (2008)

27. Wang, JR: Shannon wavelet regularization methods for a backward heat equation. J. Comput. Appl. Math. 235(9), 3079-3085 (2011)

28. Dou, FF: Wavelet-Galerkin method for identifying an unknown source term in a heat equation. Math. Probl. Eng. 2012, Article ID 904183 (2012)

29. Qian, AL: Identifying an unknown source in the Poisson equation by a wavelet dual least square method. Bound. Value Probl. 2013, 267 (2013)

30. Regińska, T, Wakulicz, A: Wavelet moment method for the Cauchy problem for the Helmholtz equation. J. Comput. Appl. Math. 223, 218-229 (2009)

31. Dou, FF, Fu, CL: A wavelet method for the Cauchy problem for the Helmholtz equation. ISRN Appl. Math. 2012, Article ID 435468 (2012)

32. Feng, XL, Ning, WT: A wavelet regularization method for solving numerical analytic continuation. Int. J. Comput. Math. 92(5), 1025-1038 (2015)

33. Cheng, W, Fu, CL, Qian, Z: Two regularization methods for a spherically symmetric inverse heat conduction problem. Appl. Math. Model. 32, 432-442 (2008) 